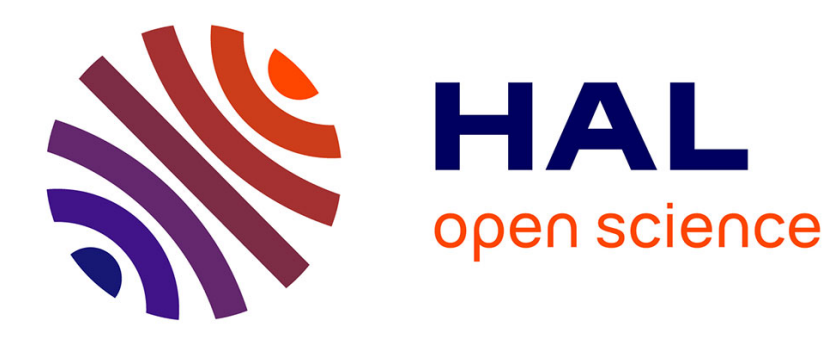

\title{
Out of nowhere: thought insertion, ownership and context-integration
}

Elisabeth Pacherie, Jean-Remy Martin

\section{To cite this version:}

Elisabeth Pacherie, Jean-Remy Martin. Out of nowhere: thought insertion, ownership and contextintegration. Consciousness and Cognition, 2013, 22, pp.111-122. 10.1016/j.concog.2012.11.012 . ijn_00778368

\section{HAL Id: ijn_00778368 \\ https://hal.science/ijn_00778368}

Submitted on 19 Jan 2013

HAL is a multi-disciplinary open access archive for the deposit and dissemination of scientific research documents, whether they are published or not. The documents may come from teaching and research institutions in France or abroad, or from public or private research centers.
L'archive ouverte pluridisciplinaire HAL, est destinée au dépôt et à la diffusion de documents scientifiques de niveau recherche, publiés ou non, émanant des établissements d'enseignement et de recherche français ou étrangers, des laboratoires publics ou privés. 


\title{
Out of nowhere: Thought insertion, ownership and context-integration
}

\author{
3 e1 Jean-Remy Martin *, Elisabeth Pacherie
}

4 Q2 Institut Jean Nicod, IEC-ENS, EHESS, CNRS, Paris, France

\author{
A R T I C L E I N F O \\ Article history: \\ Received 20 September 2012 \\ Available online $\mathrm{xxxx}$

\section{Keywords:} \\ Schizophrenia \\ Thought insertion \\ Sense of ownership \\ Sense of agency \\ Decontextualized thoughts \\ Causal-contextual information integration
}

\begin{abstract}
A B S T R A C T
We argue that thought insertion primarily involves a disruption of the sense of ownership for thoughts and that the lack of a sense of agency is but a consequence of this disruption. We defend the hypothesis that this disruption of the sense of ownership stems from a failure in the online integration of the contextual information related to a thought, in particular contextual information concerning the different causal factors that may be implicated in their production. Loss of unity of consciousness, manifested by incoherent subjective experiences is a general phenomenal characteristic of schizophrenia. This loss of coherence has been hypothesized to reflect a generalized deficit of contextual information integration not conveyed by, but related to, a target event. This deficit is manifested across many cognitive domains. We argue that it is also manifested in the process of thinking itself, resulting in causally decontextualized thoughts that are experienced as inserted thoughts.
\end{abstract}

(c) 2012 Published by Elsevier Inc.

\section{Introduction}

Imagine that you are walking in the streets of Paris and that, while walking by Notre Dame de Paris, a thought suddenly pops in your mind, say, "Kill God" (Frith, 1992). Imagine also that this thought does not feel like your own, but feels like it is somebody else's that has been inserted in your mind. For some people such an experience can feel as real as, say, their experience of the church of Notre-Dame in front of their eyes. The presence of such delusional experiences is a first-rank diagnostic symptom of schizophrenia (Schneider, 1959), a symptom called Thought Insertion (henceforth, TI). More precisely, in TI "the subject experiences thoughts which are not his own intruding into his mind. The symptom is not that he has been caused to have unusual thoughts, but that the thoughts themselves are not his" (Wing, Cooper, \& Sartorius, 1983, our emphasis; see also, Mullins \& Spence, 2003). The following reports are instances of TI:

1. I look out the window and I think that the garden looks nice and the grass looks cool, but the thoughts of Eamonn Andrews come into my mind. There are no other thoughts there, only his. [...] He treats my mind like a screen and flashes thoughts onto it like you flash a picture (Mellor, 1970, p. 17).

2. Thoughts are put into my mind, like 'Kill God'. It is just like my mind working, but it is not. They are not my thoughts. They belong to this guy, Chris. They are his thoughts (Frith, 1992, p. 66).

3. [S] he said that sometimes it seemed to be her own thought 'but I don't get the feeling that it is'. She said her 'own thoughts might say the same thing', 'but the feeling isn't the same', 'the feeling is that it is somebody else's' (AllisonBolger, 1999, \#89).

\footnotetext{
* Corresponding author. Address: Institut Jean-Nicod, Département d'Etudes Cognitives, Ecole Normale Supérieure, Université Pierre et Marie CURIE, 29 rue d'Ulm, 75005 Paris, France. Fax: +33 144322699.

E-mail address: jeanremy08@gmail.com (J.-R. Martin).
} 
As both the above definition and these examples suggest, prima facie TI seems to affect at least two phenomenal properties that normally accompany our episodes of thinking: first, the feeling or sense that our thoughts belong to us-sense of ownership (henceforth, SoO)-and, second, the feeling or sense that we are the causal generator of our thoughts-sense of agency (henceforth, SoA).

In trying to explain TI, we are confronted with several issues. First, we need to determine whether TI is best characterized as primarily a disorder of the sense of thought agency, a disorder of the sense of thought ownership or a disorder reflecting a disruption of both the sense of agency and the sense of ownership for thought. Second, as pointed out by Gallagher (2004), patients do not experience all their thoughts as inserted thoughts. An account of TI should therefore also explain its episodic nature. Third, inserted thoughts are not just negatively characterized by loss of the SoA and/or the SoO, but also positively by an attribution to an external entity postulated by patients to be the actual possessor or initiator of these special thoughts (e.g., ". . . the thoughts of Eamonn Andrews come into my mind", Mellor, 1970, our emphasis). ${ }^{1}$

With respect to the first issue, TI is often viewed as reflecting a disturbance of the SoA rather than of the SoO. Following Sousa and Swiney (2011), we call this view the standard approach. In Section 2, we will explain why we think this approach is inadequate. In the following sections, we will argue for an alternative view according to which TI essentially reflects a deficit of the SoO. We will propose the hypothesis that during the formation of some occurrent thoughts the causal factors (what we call the causal-contextual information) that produce them are not dynamically integrated by the system. As a result, these thoughts seem to come out of nowhere (we will argue that this constitutes the phenomenological basis of TI). Before presenting our main hypothesis (Section 4), we will review empirical evidence showing that schizophrenia patients present systematic integration deficits (Section 3). Finally (Section 5), we will discuss how, on this hypothesis, further distinctive features of TI could be accounted for.

\section{The standard approach criticized}

According to the standard approach, TI reflects a disturbance of the SoA for thoughts but leaves intact the SoO. One can identify two main motivations for this approach and its denial that in TI the SoO is disturbed. One motivation for this approach comes from the very influential role played by the comparator model of positive symptoms in schizophrenia proposed by Christopher Frith (1992). The second motivation comes from the inseparability thesis.

The comparator-model was originally developed to explain motor actions (e.g., Sperry, 1950). Chris Frith (1992), however, invoked it to explain delusions of control - where agents experience their own actions as controlled by some external entity - as well as several other delusions of passivity in schizophrenia, including TI (see also Campbell, 1999). According to the comparator-model, delusions of control reflect an impairment of the sense of agency for actions but leaves intact their sense of ownership for the movements that constitute these actions: patients with delusions of control claim that some external force controls their movements but do not deny that the movements thus controlled are movements of their own body. ${ }^{2}$ Frith (1992) assumed that thoughts could be analyzed as inner speech and hence could be treated as an action and applied the comparator model to thought insertion. Over the last 20 years, the comparator model of delusions of control has become far more sophisticated and has received significant empirical support. However, during the same period, the extension of the comparator-model from action to thought has been largely criticized ${ }^{3}$ and even the current partisans of the standard approach usually reject the comparator-model for TI (e.g., Gallagher, 2004; Peacocke, 2007; Proust, 2009; Young, 2008). Therefore, in the remainder of this section we will concentrate on the second motivation.

The second main reason motivating the standard view is the claim made by several authors that the so-called inseparability thesis is not challenged by TI and that therefore the SoO should not be disrupted in TI (see, e.g., Stephens \& Graham, 2000; Young, 2008). The argument from the inseparability thesis runs as follows. First, the inseparability thesis says that if I am introspectively aware of a thought I am de facto experiencing this thought as occurring within my own psychological boundaries, that is, in my own subjectivity: "By being introspectively aware of (my) thoughts [...], I am, in virtue of this, aware of what constitutes the boundaries of my mental activity (what amounts to my subjective experience)" (Young, 2008: 849). In other words, introspective awareness (of thoughts) is inseparable from the sense of subjectivity, understood

\footnotetext{
${ }^{1}$ Gallagher also points out the need to explain why inserted thoughts seem circumscribed to specific contents. This point will be not addressed here, however.

${ }^{2}$ Both the SoA for motor actions and the SoO for our body depend on the integration of multiple factors. On the one hand, the SoA is thought to rely on factors like the sensory attenuation of proprioceptive reafferences from voluntary movements, the perceptual effects of our actions and whether these effects were expected or not, the efferent signals linked to action initiation, the intention(s) preceding voluntary actions, the sense of effort experienced when executing an action and, finally, the control we have on the ongoing action (Pacherie, 2010). On the other hand, the SoO results from the multisensory integration of inputs coming from different modalities (e.g., from vision and proprioception) (Ehrsson, 2012). There is a partial overlap between the factors that determine the SoA and those involved in the $\mathrm{SoO}$ and, in ordinary situations and some neurological conditions, such as somatoparaphrenia, there is a close interplay between the SoA and the SoO (Jeannerod \& Pacherie, 2004, de Vignemont, 2009). Nonetheless, we can find striking dissociations between these two feelings, with a preserved SoO despite an impaired SoA, both in schizophrenic patients (Frith, 2005) and in healthy subjects (Kalckert et al., 2012). This indicates that the SoA may not be necessary for the SoO. In addition, a recent study with healthy subjects provides evidence that the presence or absence of a SoA over an action does not modulate the SoO for the relevant body part - i.e., the SoO is not stronger when the SoA is present than when it is absent -, and also that, in contrast, the presence or absence of a SoO for a particular body part modulates the SoA over the relevant action - i.e., the SoA is stronger when the SoO is present than when it is absent - (Kalckert \& Ehrsson, 2012). This suggests that the SoO may be more crucial for the SoA than the SoA for the SoO.

3 See for instance, Bortolotti \& Broome, 2009; Gallagher, 2004; Proust, 2008; Synofzik, Vosgerau, \& Newen, 2008a; Synofzik, Vosgerau, \& Newen, 2008b; Vosgerau \& Newen, 2007. Frith (2012) also recognizes that the comparator approach does not provide a plausible account of TI.
} 
as the sense that my thoughts are localized within the boundaries of my inner space and therefore private. Second, the inseparability thesis identifies (collapses) the SoO with the sense of subjectivity: "my awareness has inherent and inseparable subjectivity such that I cannot be aware of a thought without being likewise aware that it is my thought" (Young, 2008: 849). In other words, the sense of subjectivity is itself inseparable from the sense of ownership. Let us call the first inseparability claim (I1) and the second (I2).

Now, schizophrenic patients are introspectively aware of their inserted thoughts. Therefore, according to (I1) they experience these inserted thoughts as occurring within the boundaries of their own subjectivity, and, according to (I2), in virtue of this subjectivity, they also experience these inserted thoughts as their own. Therefore, TI cannot express a disturbed SoO; instead it should manifest a disturbed SoA: patients do not experience some of their thoughts as a product of their own agency (this is precisely the claim of the standard approach).

We think that (I2) is unwarranted and that it is wrong to identify the sense of ownership with the sense of subjectivity. First, it should be noted that unless one can produce independent reasons for thinking that the sense of ownership is preserved in TI, the argument is simply begging the question in favor of (I2). In addition, the phenomenology reported by patients suffering from TI seems to provide evidence against (I2). Patients with TI strongly feel inserted thoughts as not being their own: "the subject experiences thoughts which are not his own intruding into his mind. The symptom is not that he has been caused to have unusual thoughts, but that the thoughts themselves are not his" (Wing et al., 1983, quoted by Mullins \& Spence, 2003: 296, our emphasis). Indeed, what appears to be very troubling with TI is the fact that some introspected thoughts that occur within the subjective space of patients are not felt as being their own (Bortolotti \& Broome, 2009). If anything, TI seems to provide evidence that having a sense of subjectivity for a thought is not a sufficient condition for having a SoO for this same thought.

Second, there is also evidence, independently of TI cases, for the separability between subjectivity and ownership. Thought broadcasting is another positive symptom of schizophrenia, where patients "[...] experience [their] thoughts as escaping silently; [these] may or may not be available to other people" or "thoughts are perceived as leaving the subject's head/mind" (Pawar \& Spence, 2003: 288, ours emphasis) ${ }^{4}$. In other words, in thought broadcasting, patients experience their own thoughts escaping from their head and being broadcast, or available, to others. In thought broadcasting, therefore, patients have introspective access to their broadcasted thoughts, but these thoughts are not experienced as localized within their own psychological boundaries. The experience of these patients is thus a counter-example to (I1). Yet, these patients experience their broadcasted thoughts as being their own thoughts. Despite lacking a sense of subjectivity for their broadcasted thoughts, they retain a sense of ownership for these thoughts. Hence, broadcasted thoughts are also counter-examples to (I2).

To sum up, the identification of the SoO with the sense of subjectivity is not a necessary move. On the contrary, evidence from both TI and thought broadcasting suggest that they can dissociate and that the inseparability thesis is incorrect.

Another general objection to the standard approach ${ }^{5}$ concerns its coarse-grainedness. The standard approach characterizes TI as involving a disturbed SoA together with a preserved SoO, but cannot explain what distinguishes TI from other cases where people lack a SoA for a thought yet retain a SoO for it. It therefore fails to accurately capture its unique phenomenology.

First, the standard approach cannot readily account for the difference between TI and the normal case of unbidden thoughts (hereafter, UT). UTs are unwilled thoughts that "strike us unexpectedly out of the blue; and thoughts that run willy-nilly through our heads" (Frankfurt, 1976: 240). We lack a SoA for UTs, insofar as we do not experience ourselves as the causal source or causal generator of these thoughts, and yet we retain a SoO for them. But then, the standard theory seems bound to characterize both UTs and TI in the same way - the absence of a sense of agency in the presence of a sense of ownership. It cannot account for the difference between the relatively normal experience of unbidden thoughts and the pathological experience of thought insertion. ${ }^{6}$ In addition, the standard approach also lacks the resources needed to differentiate between TI and influenced thinking, another symptom where the SoA is disturbed but not the SoO. In influenced thinking "the patients OWN thoughts... are being controlled or influenced by an outside force" (Koehler, 1979; quoted by Mullins \& Spence, 2003: 297). Once again, if TI only affects the SoA, how do we explain the difference between TI and influenced thinking? ${ }^{7}$

The difficulties the standard approach is confronted with make us prefer a second option. In what follows we will defend the view that TI reflects a basic deficit of the SoO and that the loss of SoA for inserted thoughts is but a consequence of this primary deficit. We propose that the SoO for thoughts depends on online dynamical processes of causal-contextual information integration and that the disturbance of the SoO in TI can be explained by a disruption of these information integration processes. We start with a review of the clinical and experimental evidence showing that schizophrenic patients manifest a generalized deficit of integration information (especially of the contextual information) in many, if not all, cognitive domains.

\footnotetext{
${ }^{4}$ Note that thought broadcasting, while perhaps the most characteristic, is not the only symptom whereby patients feel their thoughts leaving their mind. In thought withdrawal, for instance, patients feel that some of their thoughts are withdrawn by an external agency such that their own thoughts are felt as leaving their head (Koehler, 1979; Pawar \& Spence, 2003).

5 The following remarks apply to the standard approach in general, whether inspired by the comparator-model or not.

${ }^{6}$ For Gallagher (2004), what differentiates UT from TI is the attributive side of the latter where thoughts are attributed to an external agency. However it will be shown in the last section that this attributive process is not systematic and that when it occurs it is as a result of the loss of the SoO in TI.

7 Sousa and Swiney (2011) distinguish between two notions of SoA. This seems to give them the tools to differentiate between the two symptoms.
} 


\section{Schizophrenia: the fragmented mind}

\subsection{Gestalt processes in schizophrenia}

Consciousness manifests itself as an integrative experience. Unity and coherence seem to be basic characteristics of subjective experiences. Our perceptual experiences as well as our thought experiences do not appear disparate, sparse or incoherent but deeply harmonious, unified and connected. However in some pathological circumstances like schizophrenia this unity of consciousness is lost. Schizophrenia is characterized phenomenally by very chaotic subjective experiences. Patients experience the outside world as well as their inner mental world as deeply fragmented (Silverstein \& Uhlhaas, 2004; Uhlhaas \& Silverstein, 2005).

Both clinical and experimental data suggest that patients have impaired Gestalt organizational processes. We first present these impairments in the perceptual domain, namely in perceptual organization: the ability of perceptual systems to form coherent and global representations of perceptual events beyond their elemental parts through some organizational rules, e.g., proximity, similarity, closure and good continuation (Wertheimer, 1923).

Ariety describes the experience of one patient in the following way: "She remembered that she could not look at the whole door. She could only see the knob or some corner of the door. The wall was fragmented into parts" (Ariety, 1962, p. 85, quoted by Silverstein \& Uhlhaas, 2004: 264). Chapman reports another patient's own description of their visual experience: "Everything I see is split up. It's like a photograph that's torn in bits and put together again. If somebody moves or speaks, everything I see disappears quickly and I have to put it together." (Chapman, 1966, p. 229, quoted by Silverstein \& Uhlhaas, 2004: 264).

Many experimental studies have shown that patients have perceptual organization deficits (for a review, see Uhlhaas \& Silverstein, 2005). In a famous study, Place and Gilmore (1980) had schizophrenia patients and healthy controls count the number of lines in tachistoscopically presented arrays comprising two to six horizontal or vertical lines at the points of an imaginary hexagon. The lines were either coherently organized (e.g., only vertical or only horizontal lines) or randomly mixed. Their hypothesis was that patients would have impaired Gestalt organizational processes, and they predicted that they would be worse than control subjects in the "coherent" condition but better in the "mixed" condition at counting the lines. These two predictions were confirmed. Later studies have replicated and extended these results, showing, for instance, that grouping is also impaired in the auditory modality (Silverstein, Matteson, \& Knight, 1996) and that other perceptual organization processes, such as closure, are also disturbed (Doniger, Silipo, Rabinowicz, Snodgrass, \& Javitt, 2001; Keefe \& Kraus, 2009). To sum up, both clinical and experimental data confirm that the perceptual world of patients is subjectively fragmented and present basic deficits in the perceptual organization processes that normally bind elements into a context-appropriate coherent whole.

\subsection{Schizophrenia and the contextual information integration deficit hypothesis}

The fact that patients present perceptual organization disturbances suggests an information integration deficit: they fail to coordinate (i.e., bind together) different elements related to a single current particular event in a coherent way (Silverstein \& Phillips, 2003, Uhlhaas, Haenschel, Nikolić, \& Singer, 2008; Uhlhaas \& Singer, 2006, 2010). More specifically, patients appear to be impaired at coordinating the surrounding contextual information related to a target event with the information strictly conveyed by the content of this target. Such a contextual information integration deficit modifies the quality of the final outcome of a processed target (Bazin, Perruchet, \& Hardy-Bayle, 2000; Silverstein \& Phillips, 2003, Silverstein \& Uhlhaas, 2004; Uhlhaas \& Silverstein, 2005). Whether contextual information is correctly integrated or not will not change the informational content strictly conveyed by the target state; however, it will modulate the salience, the valence, or the relevance of the target event, and thus its phenomenological quality.

As pointed out by Uhlhaas and Silverstein (2003), Gestalt theorists did not conceive of Gestalt laws as applying only to perceptual organization in vision, but as general laws governing cognitive and brain processes. These Gestalt conceptions find an echo in current theories of cognitive function and brain organization. Besides perceptual grouping, many cognitive functions, such as attention-dependent stimulus selection, subsystem integration, working memory, and consciousness, depend on contextual coordination between and within streams of processing. Recent theories in cognitive neuroscience have proposed that neural oscillations and their synchronization may represent a versatile functional mechanism to realize flexible communication within and between cortical areas (Singer, 1999; Uhlhaas \& Singer, 2006, 2010; Uhlhaas et al., 2008). In line with this conception, Uhlhaas and Sylverstein propose that "abnormal perceptual organization in schizophrenia is one manifestation of a larger disturbance in the combining of context-related stimuli" (2003: 14). Uhlhaas et al. (2008) further suggest that this larger disturbance may be explained by the widespread deficit in the generation and synchronization of rhythmic activity schizophrenia is associated with. There is indeed empirical evidence of contextual information integration deficits across many cognitive domains in schizophrenia.

First, in the perceptual domain the contextual information integration deficit is already present at very low levels. The specific response of a neuron to a particular stimulus can normally be amplified or reduced (i.e., modulated) according to the (level of) stimulation the neighboring neurons receive (representing the contextual information of the target neuron). These optimizing processes of reduction (or amplification in other cases) of signals belong to the so-called gain control mech- 
anisms "that allow sensory systems to adapt and optimize their responses to stimuli within a particular surrounding context” (Butler, Silverstein, \& Dakin, 2008: 41). Such optimizing processes are partially disturbed in schizophrenia patients (e.g., Butler et al., 2005). Second, patients have impaired abilities to process contextual information related to linguistic stimuli. Several experiments have shown that, compared to controls, patients integrate to a lesser degree the semantic context related to target words or sentences (Bazin et al., 2000; Passerieux, 2004). Third, some authors have proposed that the autobiographical and episodic memory impairments ${ }^{8}$ of patients could result from a deficit in the integration of the contextual elements related to the target event to be memorized during the encoding process (Danion, Rizzo, \& Bruant, 1999; Passerieux, 2004). Four, contextual information integration deficits have also been invoked to explain the difficulties encountered by schizophrenia patients in correctly identifying the intentions, beliefs and desires of others (Chambon et al., 2011; Passerieux, 2004). Finally, executive dysfunctions in schizophrenia appear to reflect a specific problem with contextual control. The difficulties they present in organizing or selecting appropriate actions in relation to internal goals have been empirically shown to result from an inability to take into account (perceptual) contextual information in order to select the appropriate action among competing options (Chambon et al., 2008).

The presence of a contextual information integration deficit across many cognitive domains in schizophrenia patients could therefore explain the fragmentation of their subjective experience (Phillips \& Silverstein, 2003; Silverstein \& Uhlhaas, 2004; Uhlhaas et al., 2008).

We propose that contextual information integration also plays an essential role in the process of thinking itself. That is the coordination of the contextual information related to a thought with its content would be essential in order to experience coherent and unified episodes of thinking. The specific kind of contextual information we are concerned with consists of the causal factors that trigger and/or constrain such episodes. A breakdown of these coordination process(es) would result in our thoughts appearing abnormal, incoherent and fragmented. In the next section, we describe these different causal factors and show how TI may stem from a deficit in the integration of these factors.

\section{Inserted thoughts as decontextualized thoughts}

\subsection{Where do our thoughts come from?}

A variety of internal and external of factors can trigger thoughts or constrain their contents. Typically, when we have a thought, we do no just have access to its content. We can also have access to surrounding information about what triggered this thought, although this information may sometimes be difficult or even impossible to retrieve (see below). We refer to this information as the causal factor(s) of thoughts and we argue that these factors constitute a specific kind of contextual information, related to, but not conveyed by, the strict content of this thought. We will argue that when, in an episode of thinking, this causal-contextual information is not properly integrated, the phenomenology associated with the thought, but not the meaning of its strict content, is affected. First, however, let us consider (some of) the factors that can trigger our thoughts.

\subsection{External factors}

Very often thoughts are driven by external factors. That is, one or many specific events in the world trigger the thought you currently have. For instance, while looking through the window I see a bird and I think: "yet another pigeon!" Such instances of externally-driven thoughts are very common.

\subsection{Internal factors}

Our thoughts can of course also be triggered by purely internal events: internally-driven thoughts. The cases of "thought association" are obvious instances. For example, while I am thinking about something (e.g., about pigeons), this thought triggers in me some memories (e.g., of having seen huge numbers of pigeons in Venice), which, in turn, generate some further thoughts (e.g., that pigeons are a threat to monuments).

Furthermore, thoughts can also be selected as a function of some internal goals (e.g., problem solving, action planning etc.). ${ }^{9}$ Thought selection in relation to internal goals involves the deployment of specific executive processes (Koechlin, Ody, \& Kouneiher, 2003; Koechlin \& Summerfield, 2007). These executive processes monitor, inter alia, the current context and your memory. Imagine you have just finished giving a talk about, say, the co-evolution of pigeons with their urban environment. Now comes the question period. You answer a first question and are then asked a second question almost identical to the first one. Getting this second, almost identical question can make you suspect that your first answer was not clear enough. So, the thoughts that will be selected for your second answer should take into account this current context and the words used for the first response. In other words, your worries about the clarity of your first answer, "define a new episode with its own 'episodic' control signals (or rules), which have to be integrated with those owing to sensorimotor and contextual control in the

\footnotetext{
${ }^{8}$ For an overview of the memory impairments in schizophrenia see for instance, Harvey \& Sharma, 2002

${ }^{9}$ For some authors, these are instances of mental-actions where thoughts are not passively but actively produced (Peacocke, 2007, 2008; Proust, 2008).
} 
service of action [or thought] selection" (Koechlin \& Summerfield, 2007: 230). Instead of simply repeating your initial answer, you try to formulate a, hopefully, clearer answer.

\subsection{Further modulating factors}

A thought originally externally- or internally-driven can be modulated by supplementary (internal or external) factors eventually determining what will be the content of a thought. Take the thought in the first example given above, namely while looking through the window I see a bird and I think: "yet another pigeon!", as the 'prototypical' thought the subject would have while looking through the window and seeing a pigeon perching on a branch right in front of the window, all other things being equal. Then the following list contains (at least some of) the factors that can modulate and possible change that prototypical thought.

I. Perceptual constraints The current perceptual conditions can also affect your thoughts. For instance, suppose I see the same bird while looking through a very dirty window, the thought "yet another pigeon!" may be replaced by the thought " is it a pigeon or a blackbird?".

II. Situational constraints: similarly, according to the current situational conditions your thought could also be different: in my room in Toronto, while looking through the window I see a bird and I think: "what! Even here there are pigeons!".

III. Doxastic background constraints: our thoughts are always generated within a personal background of beliefs and knowledge. Suppose, for instance, that I believe that in Toronto there are no pigeons, only turtledoves. While looking through the window I see a bird and I think: "Haaaa... here's a turtledove!". This particular thought is triggered by the view of the pigeon. The set of possible thoughts your system can produce is a function of your knowledge and set of beliefs. With a different doxastic background, the thought would have been different ${ }^{10}$. For instance, if I am very ignorant about birds, then while seeing a bird through the window, I could maybe think, "yet another pigeon!"; but I could not think, as a bird-watcher would, "yet another Rock pigeon!".

IV. Immediate internal constraints: a particular thought can also depend on the thought(s) that just precede it. My thought(s) about a specific perceptual object may differ according to my immediately preceding thought(s): I am thinking that golden eagles are endangered, and, while looking through the window I see a bird and think: "at least there will always be pigeons!"

V. Memory constraints: a thought about a specific stimulus or an internal event can depend on a particular memory context. For instance, while looking through the window I see a bird and I think: "I must not forget to feed the birds tonight".

VI. Emotional constraints: your current affective state can also influence the content of your thought. For some reason, today I am particularly happy and seeing a bird through the window I think: "Actually, pigeons are not so ugly!".

VII. Volitional constraints: the subject has the possibility to reject the thought(s) she currently has. That is to say, she can exert metacognitive control over her thought(s) and decide whether to go on with it or not (sometimes, of course, it is not as easy as we suggest). Suppose I am writing a paper, see a bird through the window and start thinking about pigeons. I may exert metacognitive control to abort this thinking about pigeons and go back to my writing.

In what follows we will try to show that TI may result from an integration deficit of some of these external, internal and modulating factors.

\subsection{Causal coherence determines SoO}

Our "stream" of thinking (as well as our stream of consciousness in general) appears unified and coherent and strange intrusions or serious incoherence are relatively infrequent. What are the processes that underlie this 'cohesive' phenomenology? We just explained that a thought does not arise out of nowhere; rather a number of factors contribute to its emergence. The integration of these causal-contextual factors with the content of thoughts is necessary to obtain a cohesive phenomenology within our episodes of thinking. However, this coherence is a form of causal coherence rather than semantic coherence. A specific thought will be said to be causally coherent if the system integrated its causal source(s) so that the presence of this thought within our stream of consciousness will feel "natural" and normal, independently of its semantic content. This causal coherence determines a specific phenomenology of coherence that does not depend on the (semantic) content of thoughts but on some functional processes that underlie the mechanisms of thought production. In contrast, semantic coherence depends on the information conveyed by the thoughts. It depends, inter alia, on the degree of match between the semantic content of the current thought and the semantic expectations/predictions of what its content would be based on the semantic contents of the preceding thoughts (see below). However, causal coherence may or may not co-occur with semantic coherence.

\footnotetext{
10 Of course, all thoughts are generated within a doxastic background. Nevertheless the crucial point is the weighting of the different factors within the current situation. So, the doxastic background will have more or less weight in the determination of the current thought according to the current situation.
} 
Consider first the case in which the two kinds of phenomenology co-occur. For instance, when you are running an inner soliloquy about a specific subject matter (e.g., solving a math problem), each particular thought is, at least partially, constrained by (usually implicit) semantic expectations (or semantic predictions) derived from the preceding thought(s). ${ }^{11}$ In this case causal coherence and semantic coherence co-occur. More precisely, during such an inner soliloquy the integration process of causal-contextual information integrates or coordinates each previous thought with the next thought, which, in this case, was precisely triggered by this preceding thought (what we called the immediate internal constraints). The phenomenal output of this coordination process is a causal coherence in that the presence of the upcoming thoughts feels normal. In addition, the semantic predictions of the upcoming thoughts formed upon the preceding thoughts provide a phenomenology of semantic coherence. This is phenomenally manifested as follows: when your current thoughts match the predictions derived from the previous thoughts, their semantic contents are not as salient as when there is some mismatch. Read the following sentence: "The election of B. Obama was a great historical moment, since he was the first president of the United States to be white". You were probably surprised by the last word of this sentence and its unexpectedness made it very salient. Experimentally, when subjects have to identify the sense of a target word, they are faster to recognize its sense if it was preceded by a close semantic context (Harvey \& Sharma, 2002; Passerieux, 2004). Physiologically, this priming effect is manifested by a reduction of the $\mathrm{N}^{2} 00^{\prime}$ amplitude, when the word is preceded by a close semantic context. The N400 is an event-related potential "arising in all linguistic situations, between 250 and $450 \mathrm{~ms}$ after target word presentation; its amplitude changes according to the facility to access at word meaning" (Passerieux, 2004). This process is disturbed in schizophrenia patients, who show a reduced priming effect, physiologically expressed by a smaller reduction of the N400 when the target word is preceded by a close semantic context. To recap, by a kind of semantic priming effect one thought blends smoothly into the next and they appear to form a coherent sequence. Inversely, when the semantic content of a thought does not match our expectations, it automatically becomes salient and appears incoherent or incongruent with respect to our preceding thoughts.

Take now the case in which the phenomenology of causal coherence and the phenomenology of semantic coherence come apart. While you are performing an activity (e.g., exercising at the gym) and thinking about your current performance, suddenly the thought "I must not forget my laptop for tonight's meeting" pops in your mind. In this case, the information conveyed by the thought (i.e., its semantic content) will appear salient to the extent that it was not expected or predicted from the preceding thoughts. Nonetheless, the presence of this thought does not feel abnormal. Why? The reason is that in this particular case, the system integrated the (implicit or explicit) memory factor, namely your prospective memory of tonight's meeting, that triggered or constrained the content of your thought (what we called the memory constraints). The factor could also have been a perceptual event (i.e., what we called an external factor), such as your glimpsing your laptop through the open door of the locker room. This perceptual event would have been dynamically coordinated (i.e., integrated) with your occurrent thought with the result that, once again, the presence of the thought would not have seemed abnormal (even if you were unable to retrieve this perceptual information later).

To recap, to produce phenomenal causal coherence the system must integrate the causal source(s) (i.e., the causal-contextual information) of thoughts with the thoughts themselves. Now, we propose that the SoO for thoughts directly follows from the production of phenomenal causal coherence (and not from semantic coherence). The establishment of causal coherence is what prevents the irruption of a sense of non-ownership or dis-ownership. ${ }^{12}$ When this integration process is disrupted, a thought, while occurring, will be disconnected from its causal source(s). It will then be experienced as coming out of nowhere. Imagine now that you experience some of your thoughts as coming out of nowhere; their presence will likely feel strange and abnormal so that your sense of ownership for these thoughts could be seriously disturbed. We suggest that this is precisely what happens in TI. In other words, we hypothesize that in patients with TI, the processes responsible for the online integration or coordination of the causal-contextual information pertaining to a thought with the thought itself is impaired. That is to say, the link between causal context and thought is not dynamically maintained. As the result, these thoughts would be experienced as coming from nowhere, and we would lack a SoO for them. In our view then, considering inserted thoughts as decontextualized thoughts constitutes the better way of describing the phenomenological substrate of thought insertion.

In this view, the SoO for thoughts depends essentially on the integration of relevant causal-contextual information with the occurring thought(s). This causal information may (but need not) include conscious causal factors (e.g., voluntarily produced thoughts). Additional factors, such as logical or semantic coherence may modulate the strength of the SoO. Nevertheless, we claim that causal integration is a necessary and sufficient condition for ownership, so that if it is absent the SoO will be undermined.

But now what could be the functional impairment responsible for the disturbed integration of causal-contextual information in the production of episodes of thinking?

\footnotetext{
11 The system of course also integrates other information, as the broader context in which the soliloquy is taking place. That is about what it is (i.e., the "superordinate task" the subject is executing), such as the solving of a mathematical problem. This broader context will drive the general line of your soliloquy, selecting the more appropriate thoughts according to (at least) the current situation. In this respect, if you are fully concentrated on your problem, the sentences you will silently utter will be coherent with the superordinate task. Other things being equal, a sentence such as "Pythagoras was not right about this" will appear coherent with your solving problem task, but the sentence "this bread is delicious" will not.

12 The phenomenology of thought-ownership is difficult to characterize positively. It is not a vivid and highly salient part of our experience of thinking; rather, it is better described as recessive, occupying the margins of consciousness and involving a diffuse and harmonious sense that our thoughts are part of some harmonious flow and do not come out of nowhere. In addition, it is difficult to decide if TI is phenomenally characterized only by an absence of ownership (i.e., non-ownership) or more specifically by the presence of a negative sense of dis-ownership. However, these issues are not primordial for the present paper, so they are postponed for another occasion.
} 


\subsection{Abnormal causal coherence: a working memory deficit?}

The deficit of perceptual binding between the contextual information and the stimulus in schizophrenia (see Section 3) can be explained by deficits in both "postattentive working-memory-based linkages of features across time" and "preattentive concurrent modulation from surrounding stimulus features across space" (Uhlhass \& Silverstein, 2005: 110). However, to the extent that the conscious flow of thoughts is essentially, though not exclusively, a temporal phenomenon, the disturbed function is likely to be a function centrally involved in the linking of features across time. So, we suggest that the integration deficit of causal-contextual information in the process of thought production is principally the result of working memory impairments.

We can roughly define working memory (henceforth, WM) as the ability to maintain, manipulate and coordinate online a definite quantity of information for a short period of time. An efficient WM is necessary, inter alia, for the efficient online coordination of post-attentive information across time needed for the production of coherent representations of objects and events and well-unified experiences. In the case of thinking, it is very likely that actually it is WM that enable us to link (or maintain the link of) thoughts with what we called their surrounding causal-contextual information. Indeed, there is strong evidence that patients have global WM impairments, affecting its verbal as well as its visuo- and non-visuo-spatial components (Conklin, Curtis, Katsanis, \& Iacono, 2000; Gooding \& Tallent, 2004; Harvey \& Sharma, 2002, chap. 4; Lee \& Park, 2005).

Compared to healthy people, patients maintain typically less information in WM, and their performances are not improved by decreasing the delay between the presentation and the test phase in a delayed response task (Lee \& Park, 2005). These WM impairments could therefore constitute a main cause of the failure of online integration between thoughts and their causal-contextual information. As pointed out by Lee and Park (2005) the patients' WM deficits could result from disruptions at different levels or stages in the process of a given WM task since, "to perform a working memory task successfully, one has to 'encode' the target, internally represent the target, maintain the mental representation of the target while inhibiting irrelevant information, and retrieve the mental representation at the right moment" (p. 603). Interestingly, patients have deficits at almost all these sub-processes supporting WM. First, they present encoding deficits, notably in the context of memory tasks and specifically concerning the encoding of contextual information (Passerieux, 2004). Evidence shows that contextual information is not sufficiently processed at the encoding stage, and as a much less available for future processes, including the manipulation and coordination of this information within WM (Lee \& Park, 2005). Second, patients are very impaired at inhibiting irrelevant information. They tend to over-process irrelevant information at the expense of relevant information, and as a result their mind is systematically assailed by a non-structured flow of information (Fletcher, Paul \& Frith, 2009; Keefe \& Kraus, 2009; Kraus, Keefe, \& Krishnan, 2009; Moore \& Fletcher, 2012). In addition, selective attention is disrupted, which further contributes to the systematic intrusion of irrelevant information in their flow of consciousness (Harvey \& Sharma, 2002, chap. 6). Finally, schizophrenia patients present deficits in the retrieval process itself (Harvey \& Sharma, 2002, chap. 3; Lee \& Park, 2005). All these WM deficits could contribute to impeding causal-contextual information integration processes. In the first place, relevant contextual information may remain un-encoded or be insufficiently encoded. If it is encoded, it may be encoded along with irrelevant information, which may make its latter retrieval more difficult. Finally, even if all goes well at the encoding stage, the retrieval process itself may be impaired. To sum up, we argued that the WM disturbances could account for the schizophrenia patients' failure to coordinate some of their thoughts with relevant contextual information, resulting in a disturbed causal coherence and yielding decontextualized episodes of thinking for which the SoO is lacking.

\section{Thought insertion further explained}

We have argued so far that TI is best characterized as primarily a disorder of the sense of thought ownership. However, an account of TI should also explain the episodic nature of TI; i.e. why patients do not experience all their thoughts as inserted and it should explain why the disowned thoughts are attributed to some alien agency. In addition, in Section 2 we criticized the standard approach for failing to distinguish TI from related phenomena such as UT and IFT. We now turn to these further challenges and discuss how they can be addressed on the present model, starting with the third one.

\subsection{The case of unbidden thought}

While the standard approach failed to distinguish TI from other specific cases like UT and IFT, the present model provides the tools needed to distinguish them. According to our hypothesis, TI is essentially a deficit of the SoO. We can therefore say that what distinguishes that in IFT and UT, the former the SoO is preserved but the SoA is lacking. However, this answer needs further elaboration, since TI is in a way very similar to UT, i.e., in both cases thoughts seem to arise in your mind automatically or spontaneously and are experienced as unwilled and unexpected. Prima facie then, unbidden thoughts look like cases of decontextualized thoughts. Why is it that we still own these thoughts?

The intuitive answer is to say that UTs come out of the blue, because they lack semantic coherence with the thoughts that precede them, but do not come out of nowhere, because causal coherence is retained. That is to say, the causal-contextual information is integrated at the time of occurrence of the (unbidden) thought. This is what we suggested with the example of 
the man who suddenly had the thought "I must not forget my laptop for tonight's meeting" pop in his mind while exercising at the gym: the thought felt as coming out of the blue but not out of nowhere because the system integrated the (implicit or explicit) memory factor that triggered this thought (see, Section 4$).{ }^{13}$ Now, we need to detail how this works.

Imagine you tell me, "I think that Sarkozy was not a good president", and I ask you, "Why are you thinking that?". In this case there are at least two possibilities: either you are able to retrieve the causal-contextual information, say, "I just saw someone carrying a newspaper with a picture of Sarkozy on the frontpage" or you are not able to retrieve such information and you reply, "just like that, I don't know" (UT). In both cases, however the thought feels like your own thought and feels coherent. Why?

A first possibility is to insist that in UT, some causal-contextual information may still be retrievable and exploited by a fallback mechanism. Take the following example of UT: while I am attending a boring talk the thought, "I hate pigeons!", pops in my mind. Then if you ask me why I was thinking that, I could at least reply, "Because I really hate pigeons". In a case of inserted thought, this response may not be available. The suggestion would then be that in the case of UTs but not of ITs, individuals may still be able to relate the thought to elements of their personal background of beliefs and other attitudes. The unavailability of immediate causal-contextual information would account for the feeling of surprise and unexpectedness that accompany them, but a fallback mechanism of self-interpretation would insure that their ownership is not challenged. This echoes the hypothesis defended by Stephens and Graham (2000) who argue that TI results from the inability of patients to explain some of their episodes of thinking in terms of their own beliefs or desires, that is, in terms of their own self-conception: "A person denies that is the agent of a given thought because she finds that she cannot explain its occurrence in terms of her theory or conception of her intentional psychology" (2000: 162). This explanation, however, suffers from two important limitations. First, unbidden thoughts do not always easily fit one's self-conception. I may be kept wondering why on earth the thought "I really hate pigeons" came to my mind, given that I like birds in general and do not harbor any particular ill feelings against pigeons. Moreover, unbidden thoughts can also be disturbing, unpleasant and seriously at odds with one's self-conception and we should expect the kind of fallback mechanism just sketched to lead us to disown them in such cases. Second, and conversely, inserted thoughts are not necessarily outlandish; they can also be very mundane or at least not obviously in conflict with the patient's self-conception. Indeed, schizophrenia patients may acknowledge that their inserted thoughts are thoughts that could have been theirs, as in the case of this patient quoted by Allison-Bolger: "She said her 'own thoughts might say the same thing', 'but the feeling isn't the same', 'the feeling is that it is somebody else's" (Allison-Bolger, 1999, \#89). This may lead us to suspect that the preservation of the sense of ownership for UB and its loss in TI do not fundamentally depend on whether these thoughts do or do not fit our self-conception. Something more basic seems to be at stake.

A second possibility is that in UT, but not in TI, some causal-contextual information was actually integrated with the relevant thought by online contextual integration processes. However this information would not be retrievable after the time of occurrence of the relevant thought. The suggestion then is that in UT the contextual information that triggered the thought is not processed extensively enough to be explicitly retrievable by the subject later in time, but nonetheless processed to such an extent that the system integrates it with the relevant thought at the time around its occurrence. Turn back to the example of Sarkosy. Perhaps your thought was originally triggered by a glimpsed picture of Sarkosy. However the amount of processing this information received was not sufficient for it to be maintained in working memory long enough to be retrievable later when I asked you the reason for which you were thinking that. Now it is sufficient that the system maintained and coordinated the relevant causal-contextual information at the time the thought was generated for there to also occur a concurrent, albeit transient, sense of causal coherence.

Of course, different quantitative/qualitative levels of contextual information processing will have consequences for longterm integration, and so for the sense of ownership as well. The less the contextual information is processed, the less the integration will be maintained through time and the more the sense of ownership will disappear as time passes. Suppose again your (unbidden) thought was actually triggered by a briefly glimpsed picture of Sarkosy. If 1 week later, I ask you whether you still think that Sarkozy was a bad president, you could be astonished by my question and reply, "I never said that!". You could have no memory of ever having thought that and, in appropriate terms here, insist that it is not your own thought. Conversely, the more the causal-contextual information was processed the more the integration will be maintained through time and the less the sense of ownership will disappear as time passes. If your thought, "Sarkozy was not a good president", was triggered by a lively debate where your interlocutor argued, against you, that Sarkozy actually was a very good president, even several weeks later, you likely will still be prone to self-ascribe this thought.

Finally, there is evidence that subliminal information (i.e., the prime) presented before a target event can, in certain conditions, bias your behavioral response for this target (e.g., Naccache, 2006). This means that some integration between the unconscious information (the prime) and conscious information (the target) was achieved. So, a third possibility is that in UT

\footnotetext{
${ }^{13}$ One anonymous reviewer for the present paper asked whether the explanation we offer for thought insertion could be extended to the case of primary delusions. Primary delusions are usually defined as delusions which arise "out of the blue" with no morbid antecedents or as beliefs that are not preceded by any other ideas or events. If we follow these definitions, perhaps the distinction between "out of nowhere" and "out of the blue" we draw in the paper is relevant to this issue. A thought (or a set of thoughts) will be said to be coming "out of nowhere" if causal coherence is lacking (leading to a disturbed sense of ownership), and "out of the blue" if semantic coherence is lacking. So, we may hypothesize that the phenomenological side of primary delusional beliefs (i.e., their lack of coherence with the person's belief system) may be explained by a disturbed semantic coherence (resulting, e.g., from a deficit in the prediction of upcoming thoughts), while causal coherence is preserved (explaining why patients do not disown their thoughts in this case). Why primary delusions have the contents they have and why they become firmly held beliefs are other matters, that fall outside of the scope of the present paper.
} 
the causal-contextual information that triggered your thought was never consciously accessible (not just irretrievable later in time). However, the (unconscious) contextual information would still be integrated with the content of your present thought at the time of its occurrence. To put it metaphorically, in UT I may not know where the thought comes from, but my brain knows it and, as a result, I know it is mine. The three possibilities we delineated are not mutually exclusive and each may contribute to explaining the preservation of the sense of ownership in episodes of UT.

Now, in TI the concern is about thoughts that are not accompanied by a sense of ownership at the time of their occurrence. The concern is not about the question of self-ascription at longer term that engage other processes involving, inter alia, the relation between the encoding processes and the long term memory. As just shown, UTs are accompanied by a sense of ownership at least at the time of their occurrence. This is explained by the fact that (as the extreme case of subliminal priming shows) the online integration between the content of thoughts and their non-accessible or non-retrievable causal-contextual information is possible within a limited time window. So, we can argue that in TI even this very limited form of integration is not achieved. The online binding between the (retrievable, irretrievable or inaccessible) causal-contextual factors related to a thought and the thought itself is either absent or not maintained long enough to produce even a minimally cohesive phenomenology and thus support a sense of ownership.

\subsection{The episodic nature of TI explained}

A disruption of the WM could also account for the episodic nature of TI. Schizophrenia patients with TI do not experience all their thoughts as inserted (Gallagher, 2004). This episodic character of TI must be explained. WM processes support the online binding of contextual information. ${ }^{14}$ While WM processes are disturbed in schizophrenia, their failure is not complete. For instance, the intrusion of irrelevant information is very frequent but not systematic. Moreover, the amount of noise created by this intrusion of irrelevant information is not always important enough to disrupt the coordination of information or it may lead to spurious coordination of information, but coordination nonetheless. In addition, we have seen that patients present impairments at the encoding phase of the WM too and that these impairments could negatively affect the integration of the causal-contextual information (Section 4). Yet, there is also evidence that the performance of patients at this encoding phase can be improved by making the relevant information more salient and thus preventing it from being drowned in the noise produced by the intrusion of irrelevant information (Harvey \& Sharma, 2002, chaps. 4 and 5). We can therefore expect that in cases where the relevant causal-contextual information is by itself highly salient, integration may still operate.

\subsection{Sense of agency in thought insertion}

While we argue that TI is primarily characterized by a disturbed SoO, this does not mean that we take patients with TI to have a preserved SoA over their inserted thoughts. Rather, we take it that lack of a SoA is normally a consequence of lack of SoO. However, we cannot go as far as to say squarely that having a SoO over a thought is a necessary condition for having a SoA over this thought. In Section 2, we drew a threefold distinction between SoO, SoA, and sense of subjectivity and argued that they can dissociate. In particular, one can have a sense of agency over a thought, while lacking both a sense of subjectivity and a SoO for that thought, as in experiences of activity where patients feel that they control the thoughts of others (Mullins \& Spence, 2003). This phenomenon suggests that SoO is not a precondition of SoA. However, it does not seem possible to have both a sense of subjectivity and a SoA over a thought, while lacking a SoO over that thought. This suggests that when one has a sense of subjectivity over a thought, having a SoO over that thought is indeed a necessary condition for also having a SoA. Insofar as the sense of subjectivity is preserved in TI, the lack of SoO implies a lack of SoA.

\subsection{The attribution of TI to an external entity: a process of recontextualization}

There remains the problem of attribution: patients (sometimes) attribute their inserted thoughts to another (not always identified) agent, as exemplified in the following, often quoted, patient's self-report, 'I look out of the window and I think the garden looks nice and the grass looks cool, but the thoughts of Eamonn Andrews come into my mind. There are no other thoughts there, only his. [...] He treats my mind like a screen and flashes his thoughts onto it like you flash a picture' (Mellor, 1970: 17). Accordingly, we should distinguish in TI the negative sense of non-ownership, "it is not my thought", from the positive sense of alienation, "it is the thought of someone else" (e.g., Stephens \& Graham, 2000). It seems that TI not only manifests an abnormal sense of ownership but also involves an experience of alienation. Our hypothesis that inserted thoughts are decontextualized thoughts seems to explain the lack of a proper sense of ownership but not the positive attribution of agency.

Note first that while patients attribute their inserted thoughts to some alien agency, they do not systematically think of this agency as an identified human agent. Some attribute the source of their inserted thoughts to unknown agents, others to supernatural entities (God, spirits, devils...); others yet to machines such as radios or televisions. Various causal mechanisms are also reported by patients, for instance hypnosis or electrical signals (Mullins \& Spence, 2003). So, the attribution

\footnotetext{
14 Note that the idea that TI is a phenomenon involving a feeling that the thought has come out of nowhere and that this may be caused by some problem with the temporal dynamics of cognition and working memory is also suggested by Gallagher (2005).
} 
to a specific human agent seems to be one kind of interpretation among others of the source of inserted thoughts rather than a fundamental experiential characteristic of TI and cultural factors may influence patients' interpretations. We therefore propose a two-step account: first, inserted thoughts are decontextualized thoughts and this decontextualization accounts for the loss of sense of ownership characteristic of TI (i.e., accounts for the experiential part of TI); second, the attributional element in inserted thoughts is the result of an attempt at interpretation patients engage into make sense of this strange experience. Indeed, if inserted thoughts present themselves as decontextualized thoughts, then it is almost normal to search for an explanation of the reason why these thoughts are there, in your head. The different kinds of attribution that patients make could therefore be understood as attempts to recontextualize these thoughts that first seemed to come out of nowhere. The fact that patients often opt for extraordinary explanations could result in part from the extraordinary phenomenology of TI as decontextualized thoughts. Extraordinary events call for extraordinary explanations, so to speak. In addition, the loss of general coherence that affects schizophrenia patients would not make them very good judges of the weirdness of the explanation they are giving for their inserted thoughts.

\section{Summary}

We first argued that viewing the phenomenon of thought insertion in schizophrenia as merely reflecting a disruption of the sense of agency fails to adequately capture its phenomenology. We proposed instead that thought insertion primarily involves a disruption of the sense of ownership and we defended the hypothesis that this disruption stems from a failure in the online integration of the contextual information related to a thought, in particular contextual information concerning the different causal factors that may be implicated in its production. We argued that this failure could be explained by deficits in post-attentive working-memory-based processes involved in the linkage of features across time. This failure would result in very chaotic subjective experiences of thinking characterized, lacking phenomenal causal coherence. Accordingly some thoughts would be experienced as coming out of nowhere (i.e., as decontextualized thoughts) lacking even a minimally cohesive phenomenology. This would constitute the phenomenological basis of TI (i.e., sense of non-ownership). We also argued that the episodic nature of thought insertion could be explained by the fact that disruptions of the working-memory-based information integration are not systematic in nature. Finally we pointed out that the attribution of inserted thoughts to another agent is not systematic and proposed that it should be viewed as the result of an attempt to recontextualize these thoughts.

\section{Uncited references}

De Vignemont (2010) and Fletcher and Frith (2009).

\section{References}

Allison-Bolger, V. Y. (1999). Collection of case histories. Unpublished typescript.

Bazin, N., Perruchet, P., \& Hardy-Bayle, M. C. (2000). Context-dependent information processing in patients with schizophrenia. Schizophrenia Research, 45, 93-101.

Bortolotti, L., \& Broome, M. (2009). A role for ownership and authorship in the analysis of thought insertion. Phenomenology and the Cognitive Sciences, 8(2), $205-224$.

Butler, P. D., Zemon, V., Schechter, I., Saperstein, A. M., Hoptman, M. J., Lim, K. O., et al (2005). Early-stage visual processing and cortical amplification deficits in schizophrenia. Archives of General Psychiatry, 62, 495-504.

Butler, P. D., Silverstein, S. M., \& Dakin, S. C. (2008). Visual perception and its impairment in schizophrenia. Biological Psychiatry, 64(1), 40-47.

Campbell, J. (1999). Schizophrenia, the space of reasons, and thinking as a motor process. The Monist, 82(4), 609-625.

Chambon, V., Franck, N., Koechlin, E., Fakra, E., Ciuperca, G., Azorin, J. M., et al (2008). The architecture of cognitive control in schizophrenia. Brain, 131, 962-970.

Chambon, V., Pacherie, E., Barbalat, G., Jacquet, P., Franck, N., \& Farrer, C. (2011). Mentalizing under influence: Abnormal dependence on prior expectations in patients with schizophrenia. Brain, 134(12), 3725-3738.

Conklin, H. M., Curtis, C. E., Katsanis, J., \& Iacono, W. G. (2000). Verbal working memory impairment in schizophrenia: Evidence from the digit span task. American Journal of Psychiatry, 157, 275-277.

Danion, J.-M., Rizzo, L., \& Bruant, A. (1999). Functional mechanisms underlying impaired recognition memory and conscious awareness in patients with schizophrenia. Archives of General Psychiatry, 56, 639-644.

De Vignemont, F. (2010). Body schema and body image-Pros and cons. Neuropsychologia, 43(3), 669-680.

Doniger, G. M., Silipo, G., Rabinowicz, E. F., Snodgrass, J. G., \& Javitt, D. C. (2001). Impaired sensory processing as a basis for object-recognition deficits in schizophrenia. The American Journal of Psychiatry, 158(11), 1818-1826.

Ehrsson, H. H. (2012). The concept of body ownership and its relation to multisensory integration. In B. E. Stein (Ed.), The new handbook of multisensory processes (pp. 775-792). Cambridge, MA: MIT Press.

Fletcher, P. C., \& Frith, C. D. (2009). Perceiving is believing: A Bayesian approach to explaining the positive symptoms of schizophrenia. Nature Reviews. Neuroscience, $10(1), 48-58$.

Frankfurt, H. (1976). Identification and externality. In A. O. Rorty (Ed.), The identities of persons (pp. 239-251). Berkeley: University of California Press.

Frith, C. D. (1992). The cognitive neuropsychology of schizophrenia. Hillsdale: Erlbaum.

Frith, C. D. (2005). The self in action: Lessons from delusions of control. Consciousness and Cognition, 14, 752-770.

Frith, C. D. (2012). Explaining delusions of control: The comparator model 20 years on. Consciousness and Cognition, 21(1), 52-54.

Gallagher, S. (2004). Neurocognitive models of schizophrenia: A neurophenomenological critique. Psychopathology, 37(1), 8-19.

Gooding, D. C., \& Tallent, K. A. (2004). Nonverbal working memory deficits in schizophrenia patients: Evidence of a supramodal executive processing deficit. Schizophrenia Research, 68(2-3), 189-201.

Harvey, D. P., \& Sharma, T. (2002). Understanding and treating cognition in schizophrenia: a clinician's handbook. London: Martin Dunitz.

Jeannerod, M., \& Pacherie, E. (2004). Agency, simulation and self-identification. Mind E Language, 19(2), 113-146. 
Keefe, R. S. E., \& Kraus, M. S. (2009). Measuring memory-prediction errors and their consequences in youth at risk for schizophrenia. Annals of the Academy of Medicine, Singapore, 38(5), 414-416.

Kalckert, A., \& Ehrsson, H. H. (2012). Moving a rubber hand that feels like your own: A dissociation of ownership and agency. Frontiers in Human Neuroscience, 6(40), 1-14.

Koehler, K. (1979). First rank symptoms of schizophrenia: Questions concerning clinical boundaries. British Journal of Psychiatry, 134, $236-248$.

Koechlin, E., \& Summerfield, C. (2007). An information theoretical approach to prefrontal executive function. Trends in Cognitive Sciences, 11(6), 231-235.

Koechlin, E., Ody, C., \& Kouneiher, F. (2003). The architecture of cognitive control in the human prefrontal cortex. Science, 302(5648), 1181-1185.

Kraus, M. S., Keefe, R. S. E., \& Krishnan, R. K. R. (2009). Memory-prediction errors and their consequences in schizophrenia. Neuropsychology Review, 19(3), $336-352$.

Lee, J., \& Park, S. (2005). Working memory impairments in schizophrenia: a meta-analysis. Journal of Abnormal Psychology, 114, 599-611.

Mellor, C. S. (1970). First rank symptoms of schizophrenia. I. The frequency in schizophrenics on admission to hospital. II. Differences between individual first rank symptoms. British Journal of Psychiatry, 117, 15-23.

Moore, J. W., \& Fletcher, P. C. (2012). Sense of agency in health and disease: A review of cue integration approaches. Consciousness and Cognition, 21(1), 59-68.

Mullins, S., \& Spence, S. A. (2003). Re-examining thought insertion. Semi-structured literature review and conceptual analysis. The British Journal of Psychiatry: The Journal of Mental Science, 182, 293-298.

Naccache, L. (2006). Le Nouvel Inconscient. Freud, Christophe Colomb des neurosciences. Paris: Odile Jacob.

Pacherie, E. (2010). Self-agency. In S. Gallagher (Ed.), The oxford handbook of the self (pp. 440-462). Oxford: Oxford University Press.

Passerieux, C. (2004). Schizophrénie et sciences cognitives. In C. Spadone (Ed.), Les Schizophrénies (pp. 109-124). Paris: Pil.

Pawar, V. A., \& Spence, S. A. (2003). Defining thought broadcast, semi-structured literature review. British Journal of Psychiatry, 183, $287-291$.

Peacocke, C. (2007). Mental action and self-awareness (I). In J. Cohen \& B. McLaughlin (Eds.), Contemporary debates in the philosophy of mind (pp. 358-376). Oxford: Blackwell.

Peacocke, C. (2008). Mental action and self-awareness (II): Epistemology. In L. O’Brien \& M. Soteriou (Eds.), Mental action (pp. 192-214). Oxford: Oxford University Press.

Place, E. J., \& Gilmore, G. C. (1980). Perceptual organization in schizophrenia. Journal of Abnormal Psychology, 89(3), 409-418.

Proust, J. (2008). Is there a sense of agency for thought? In L. O'Brien \& M. Soteriou (Eds.), Mental action (pp. 253-279). Oxford: Oxford University Press. Schneider, K. (1959). Clinical psychopathology. New York: Grune and Stratton.

Silverstein, S. M., Matteson, S., \& Knight, R. (1996). Reduced top-down influences in auditory perceptual organization in schizophrenia. Journal of Abnormal Psychology, 105, 663-667.

Silverstein, S. M., \& Uhlhaas, P. J. (2004). Gestalt psychology: The forgotten paradigm in abnormal psychology. The American Journal of Psychology, 117(2), 259-277.

Singer, W. (1999). Neuronal synchrony: A versatile code for the definition of relations? Neuron, 24, 49-65.

Sousa, P., \& Swiney, L. (2011). Thought insertion: Abnormal sense of thought agency or thought endorsement? Phenomenology and Cognitive Science. http:// dx.doi.org/10.1007/s11097-011-9225-z.

Stephens, G. L., \& Graham, G. (2000). When self-consciousness breaks: Alien voices and inserted thoughts. Cambridge: MIT Press.

Synofzik, M., Vosgerau, G., \& Newen, A. (2008a). Beyond the comparator model: A multifactorial two-step account of agency. Consciousness and Cognition, 17, 219-239.

Synofzik, M., Vosgerau, G., \& Newen, A. (2008b). I move, therefore I am: A new theoretical framework to investigate agency and ownership. Consciousness and Cognition, 17, 411-424.

Uhlhaas, P. J., \& Silverstein, S. M. (2003). The continuing relevance of Gestalt psychology for an understanding of schizophrenia. Gestalt Theory, 25(4), 1-24.

Uhlhaas, P. J., \& Silverstein, S. M. (2005). Perceptual organization in schizophrenia spectrum disorders: Empirical research and theoretical implications. Psychological Bulletin, 131(4), 618-632.

Uhlhaas, P. J., \& Singer, W. (2006). Neural synchrony in brain disorders: Relevance for cognitive dysfunctions and pathophysiology. Neuron, 52(1), 155-168. Uhlhaas, P. J., \& Singer, W. (2010). Abnormal neural oscillations and synchrony in schizophrenia. Nature Reviews. Neuroscience, 11(2), $100-113$.

Uhlhaas, P. J., Haenschel, C., Nikolić, D., \& Singer, W. (2008). The role of oscillations and synchrony in cortical networks and their putative relevance for the pathophysiology of schizophrenia. Schizophrenia Bulletin, 34(5), 927-943.

Vosgerau, G., \& Newen, A. (2007). Thoughts, motor actions, and the self. Mind \& Language, 22, 22-43.

Wertheimer, M. (1923). Untersuchungen zur Lehre von der Gestalt: II [Studies toward the theory of Gestalt. II]. Psychologisch Feorschung, 4, 301-350.

Wing, J. K., Cooper, J. E., \& Sartorius, N. (1983). Present state examination (9th ed.). Cambridge: Cambridge University Press.

Young, G. (2008). On how a child's awareness of thinking informs explanations of thought insertion. Consciousness and Cognition, 17(3), 848-862. 